\title{
Contrast Enhancement Satellite Images: A Hybrid Solution for Cloud Removal
}

\author{
Abdelfattah \\ Elsharkawi \\ Faculty of \\ engineering, \\ Al- Azhar University \\ Egypt
}

\author{
Kamal A. \\ EIDahshan \\ Faculty of Science, \\ Al-Azhar University, \\ Egypt
}

\author{
Eman K. Elsayed \\ Faculty of Science \\ (girls), Al-Azhar \\ University, Egypt
}

\author{
Mahmoud Eltaher \\ Faculty of Science, \\ Al-Azhar University, \\ Egypt
}

\begin{abstract}
The contrast enhancement of the satellite images without producing unnatural and unclear images is an important challenge in image processing. Also, the clouds are an important issue in the real satellite image. So, this paper proposes a method to enhance the contrast of the cloudy satellite image. The proposed method relies on modifying and integrating the closest spectral fit and genetic algorithm to remove clouds and to detect the number of edges as well as the contrast relative difference. This leads to ameliorate the contrast satellite images. Final experimental results of applying the proposed method on real images taken by LandSat 8 show that it produce semi-natural looking images even if the image is cloudy.
\end{abstract}

\section{General Terms}

Genetic Algorithm, Image processing, Remote sensing.

\section{Keywords}

Contrast enhancement, satellite images, cloud removal.

\section{INTRODUCTION}

Producing a good quality images is an important requirement in several areas like remote sensing. Processing of remote sensing satellite images passes through four phases; namely the initial identification phase which is concerned with the direct detection of visual things in the images, the content differentiation phase in which visual things are classified and put into groups, the analysis phase which is concerned with identifying groups of objects that they have unique characteristics setting the boundaries that separate these groups, and finally the interpretation phase which links groups of objects that have been identified at the end of the analysis process with Natural content [1]. Producing images with good quality is called image enhancement which is part of the first phase. Image enhancement techniques can be classified into two categories; namely spatial domain methods and frequency domain methods [2]. Spatial domain methods deal directly with pixels. The values of pixel intensity are manipulated to get the best enhancement of images. In frequency domain methods, images are first transferred to the frequency domain (i.e. Fourier transform is computed first). All the enhancements are performed on the Fourier transform of the image intensity distribution function and then the inverse Fourier transform of the image are performed to get the resultant image. These enhancement operations are performed to enhance brightness or contrast of the image (the distribution of gray levels in the image). The enhancement process can be expressed as transforming image $\mathrm{I}$ to image $\mathrm{O}$ using transformation function [2].

$$
\mathrm{O}=\mathrm{T}(\mathrm{I})
$$

Many applications of image processing are widely used image enhancement techniques since the image's quality is an important for human interpretation. The important factor in any evaluation of image quality is the contrast. The difference in luminance between two adjacent surfaces is known as contrast. In other words, contrast is determined by get the difference in visual properties which makes an object distinguishable from other objects and the background. Many contrast enhancement algorithms have been developed and applied to image processing problems [3].The most popular approach to enhancing the contrast of images is histogram equalization [4]. But these traditional methods normally produce unnatural images. It is important to keep in mind that enhancement is a very subjective area of image processing. In image enhancement, genetic algorithms are applied to an image to get the new enhanced image that looks better than the original one which contains the features of its parents [5]. Previous experiments showed that genetic algorithms are very effective in the contrast enhancement and produces images with natural contrast.

The aim of this paper is to propose a method based mainly on genetic algorithm to enhance the contrast of satellite images even if they are clouds. Therefore these enhanced images are used in many applications without the need to wait until the weather is clear and non-cloudy to take new non cloudy images. Practically it's useful.

The rest of the paper is organized as follows: in section two related work will be discussed. Then section three will introduce the proposed method in details with implementation. The analysis of results and also the conclusions are presented in section four and five, respectively.

\section{RELATED WORK}

There are many methods that are used for image processing based on genetic algorithms as discussed below:

In reference [6], authors proposed a genetic algorithm with Simulated Binary Crossover (SBX) multilevel threshold. That was to segment the medical brain images precisely. The optimum multilevel threshold evaluated by maximizing the entropy. 
But reference [7] presented a genetic algorithm to demonstrate effectiveness in solving the problem of unsupervised image classification. It is one of the optimization problems in a large domain. The authors built a fitness function to use it as a classifier. That is to improve the classification accuracy and robustness of the image classification.

Also, authors in reference [8] proposed a contrast enhancement method based on a genetic algorithm. Their main contribution was to use a simple chromosome structure and genetic operators to increase the visible details and contrast of low illumination images, especially with high dynamic ranges. The approach maps each gray level of input images to another one such that the resulting image has better contrast.

Sometimes the images are covered. That may affect the intensity distribution of images and hence may give the fake interpretation of edges. So, reference [9] proposed an approach to remove clouds in Landsat-8 operational land imager (OLI) data. The algorithm was developed with independent component analysis (ICA). Within cloud-covered areas, histograms were derived to quantify changes of the reflectance values before and after the use of the algorithm. Referred to a cloud-free image, changes of histogram curves validated the algorithm. Another reference also tried to solve the cloud problem [10]. The authors proposed the approach which removes cloud-contaminated portions of a satellite image and then reconstructs the information of missing data utilizing temporal correlation of multi-temporal images. The basic idea is to clone information from cloud-free patches to their corresponding cloud-contaminated patches under the assumption that land covers change insignificantly over a short period of time.

\section{PROPOSED METHOD}

The objective of the proposed method is to improve the image quality of satellite image. So, two problems that affect the image quality of satellite images will be solved namely; clouds and cloud shadows in images and low contrast of images. The process of enhancement will do in two sequential steps which explain in more details in the next subsections. The sequential phases are to remove clouds from satellite images then enhance the contrast of these images. Most of the existing image enhancement methods are unable to enhance contrast in cloudy satellite images. The framework of the proposed method is presented in figure 1.

\subsection{The first phase is "Cloud removal"}

Sometimes, the weather is cloudy. So, applying any enhanced method is not enough without cloud removal first. That is shown in Figure 2.

So the goal of this preprocesses phase is to remove clouds from satellite images before enhancing them. The cloud removal proposed process is based on the closest spectral fit technique.

The proposed cloud removal process is described as follows.

\subsubsection{The proposed cloud pixels and cloud shadows pixels detection step}

Detecting cloud pixels and cloud shadows pixels using "Minimum Error threshold Algorithm" on the original image as described in the following figure 3 .

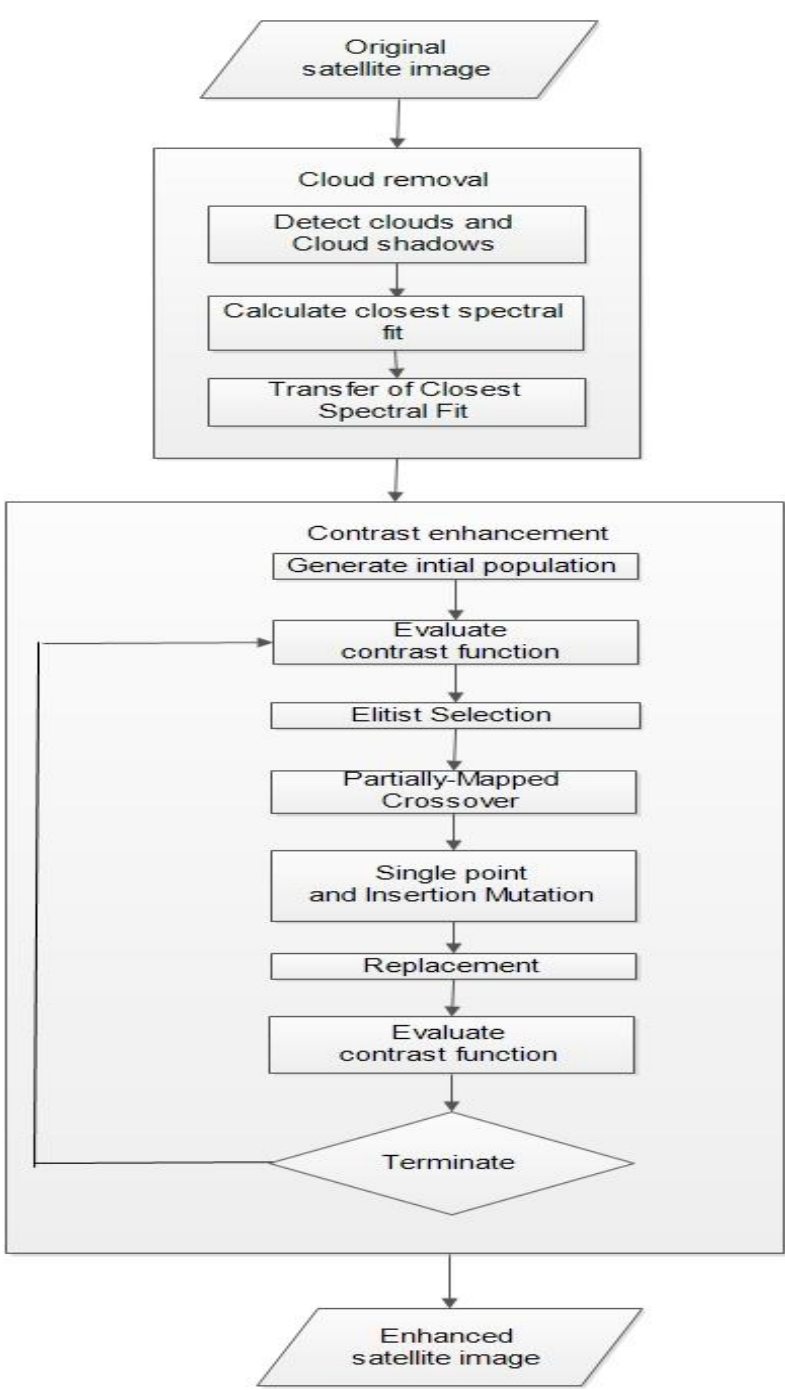

Fig 1: The proposed methodology for satellite image

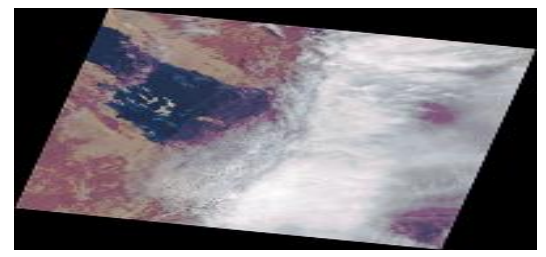

Fig 2: Applying GA in cloudy weather without cloud removal phase

\subsubsection{The proposed method to calculate the closest Spectral Fit}

In this step, the distance between each pixel and its closest pixel is determined in its individual band of the spectral space.

\subsubsection{The proposed method to transfer of closest Spectral Fit}

Having a base image that may contain cloud pixels and a corresponding auxiliary image taken for the same site but with its pixels most probably free of clouds, let's define the following:

Pixel A: the cloud pixel in the base image

Pixel a: the corresponding pixel to pixel " $\mathrm{A}$ " in the auxiliary image 
Begin

Initialize array $\mathrm{J}$ with very big values

Compute the histogram of the image intensity values Compute the relative histogram divided by size of image Walk through every possible threshold $\mathrm{T}$ (a possible threshold would lie in the interval [0...255]. $\mathrm{T}$ is interpreted as the lower boundary of the second class of pixels rather than the upper boundary of the first class.

For $\mathrm{T}$ is 0 to 255

Split the histogram at the threshold T.

Calculate histogram 1 is sub array of histogram array from index 1 to index $\mathrm{T}$ (first class)

Calculate histogram 2 is sub array of histogram array

from index $\mathrm{T}+1$ to last index of histogram array

(second class)

Compute the number of pixels in the two classes. $P 1=\sum_{i=1}^{h 1}$ histogram1(i) , where h1 is the length of histogram 1

$P 2=\sum_{i=1}^{h 2}$ histogram2 $(i)$, where h2 is the length of histogram2

Compute the standard deviations of the classes.

$$
\begin{gathered}
\text { mean } 1=\frac{1}{P 1} \sum_{i=1}^{T} \text { histogram } 2(i) * i \\
\text { mean } 2=\frac{1}{P 2} \sum_{i=1}^{256-T} \text { histogram } 2(i) * i \\
\text { sigma } 1=\sqrt{\frac{1}{P 1} \sum_{i=1}^{T}(i-\text { mean } 1) * \text { histogram } 1(i)^{2}} \\
\text { sigma } 2=\sqrt{\frac{1}{P 2} \sum_{i=1}^{256-T}(i-\text { mean } 2) * \text { histogram } 2(i)^{2}}
\end{gathered}
$$

Compute the criterion function.

$\mathrm{J}(\mathrm{T})=1+2 *(\mathrm{P} 1 * \log (\operatorname{sigma} 1)+\mathrm{P} 2 * \log ($ sigma 2$)) \ldots$

$$
-2 *(\mathrm{P} 1 * \log (\mathrm{P} 1)+\mathrm{P} 2 * \log (\mathrm{P} 2))
$$

endfor

Find the minimum of $\mathbf{J}$ (optimal Threshold is equal to the minimum of $\mathbf{J}$ )

End

\section{Fig 3: Minimum Error threshold Algorithm}

Pixel b: the closest pixel in the auxiliary image

Pixel B: corresponding pixel to pixel "b" in the base image

For pixels covered with clouds and cloud shadows, find pixel $\mathrm{A}$ and its location-based one-to-one correspondence pixel "a".

Find in the auxiliary image a's most similar "b".

Replace the intensity value at $\mathrm{A}$ in the base image with the intensity value at $\mathrm{B}$ and the difference between the value of the corresponding free-pixel in "a" auxiliary image and the closest spectral pixel in the auxiliary image "b".

\subsection{The second phase is "contrast enhancement"}

This phase is based on Genetic Algorithms (GA). GA is search optimization algorithm based on the principle of natural selection. They provide systematic random search by

\begin{tabular}{|c|c|c|c|c|c|c|c|c|c|}
\hline 0 & 15 & 30 & 70 & 90 & 110 & 161 & 210 & 225 & 255 \\
\hline 1 & 2 & 3 & 4 & 5 & 6 & 7 & 8 & 9 & 10 \\
\hline
\end{tabular}

Fig 4: Chromosome structure using three operators; namely selection, crossover, and mutation. The following subsections discuss the details of the proposed genetic algorithm and as well as its operators for image enhancement.

\subsubsection{The chromosome structure}

Each chromosome is an array of length $\mathrm{n}$ of random integer numbers where $\mathrm{n}$ represents gray levels in the image. This array is sorted in ascending order. To have a good contrast, the gray levels of the image are stretched by setting the first element of the array to 0 , and the last element of the array is set to 255 as shown in figure 4 .

\subsubsection{The contrast fitness function}

The most important problem in genetic algorithms is to determine the suitable fitness function for a given set of chromosomes. It can be chosen well by experience only, so some examples are written out of the testing data, and then think about the kind of function which can be used. In this paper, the fitness function which consists of "the contrast relative difference"(CRD) is proposed, a number of edges and the overall intensity. The proposed fitness function is shown in equation (2). The proposed fitness function will take into consideration the contrast of the image without ignoring of the details of the image which is represented by the number of edges. Improving the contrast is expected to give a better view of images and also even reaching better details.

The proposed fitness function is shown in equation 2 .

Fitness $(\mathrm{x})=\log (\log (\mathrm{E}(\mathrm{I}(\mathrm{x})))) * \mathrm{n} \_$edges $(\mathrm{I}(\mathrm{x}))$

$$
\text { * CRD . }
$$

- Where

- $\quad$ Fitness $(\mathrm{X})$ denotes the fitness value of chromosome $\mathrm{X}$ and $\mathrm{I}(\mathrm{X})$ is the enhanced image.

- $\quad$ First: Number of edges.

- $\quad \mathrm{n}$-edges $(\mathrm{I}(\mathrm{X}))$ presents the number of detected edges in the enhanced image which is calculated by "the Sobel edge detector" [11].

- Second: the overall intensity.

- The sum of the intensity values of the enhanced image, is expressed by $\mathrm{E}(\mathrm{I}(\mathrm{X})$ ) which is calculated by the following equation (3).

$$
\mathrm{E}(\mathrm{I}(\mathrm{X}))=\sum_{\mathrm{x}=0}^{\mathrm{m}} \sum_{y=0}^{n} \mathrm{dh}(\mathrm{x}, \mathrm{y})^{2}+\mathrm{dv}(\mathrm{x}, \mathrm{y})^{2} .
$$

- $\quad d h(x, y)$ : sum of the horizontal intensities of the image

$$
\begin{gathered}
\mathrm{dh}(\mathrm{x}, \mathrm{y})=\mathrm{g}(\mathrm{x}+1, \mathrm{y}-1)+2 \mathrm{~g}(\mathrm{x}+1, \mathrm{y})+\mathrm{g}(\mathrm{x}+1, \mathrm{y}+1)- \\
\mathrm{g}(\mathrm{x}-1, \mathrm{y}-1)-2 \mathrm{~g}(\mathrm{x}-1, \mathrm{y})-\mathrm{g}(\mathrm{x}-1, \mathrm{y}+1)
\end{gathered}
$$

- $\quad g(x+1, y-1):$ Pixel with $" \mathrm{x}+1$ "horizontal coordinate and "y-1" vertical coordinate

- $\quad d v(x, y)$ : the sum of the vertical intensities of the image

$$
\begin{gathered}
d v(x, y)=g(x-1, y+1)+2 g(x, y+1)+g(x+1, y+1)- \\
g(x-1, y-1)-2 g(x, y-1)-g(x-1, y-1)
\end{gathered}
$$

- $\quad$ Third: the contrast relative difference (CRD)

$\mathrm{CRD}=\sum_{\mathrm{x}=0}^{\mathrm{m}} \sum_{y=0}^{n}\left|\frac{\mathrm{S}_{\text {new }}(\mathrm{x}, \mathrm{y})-\mathrm{S}_{\text {org }}(\mathrm{x}, \mathrm{y})}{\mathrm{S}_{\text {new }}(\mathrm{x}, \mathrm{y})+\mathrm{S}_{\text {org }}(\mathrm{x}, \mathrm{y})}\right|$ 
- $\operatorname{Snew}(x, y)$ : Pixel with "x" horizontal coordinate and " $y$ " vertical coordinate in the new enhanced image.

- $\operatorname{Sorg}(x, y)$ : Pixel with "x" horizontal coordinate and "y" vertical coordinate in the original image.

- $\mathrm{M}$ and $\mathrm{N}$ : are length and width of the image respectively.

- $\quad$ N.B. a log-log measure of the edge intensity is used to prevent producing unnatural images.

\subsubsection{Evaluation}

Each chromosome is evaluated by the fitness function in equation 2 .

\subsubsection{Elitist Selection}

After evaluation, the elitist selection is then used because this selection helps to ensure that will be the best chromosomes always chosen. Assuming that $\mathrm{Ps}$ is the number of chromosomes and $\mathrm{Pc}$ is the crossover probability, Ps* $\mathrm{Pc}$ individuals are selected to create the same number of individuals by the crossover operator.

\subsubsection{Partially-mapped crossover}

The partially-mapped crossover approach was used. Therefore, the number of selected chromosomes will be Ps*Pc where $\mathrm{Ps}$ is the number of population and $\mathrm{Pc}$ is the crossover probability. The partially-mapped crossover is done by map number of genes in each parent by the corresponding genes in another parent to produce two new children. Finally, each new chromosome is sorted again in ascending order to maintain the structure of the chromosome.

\subsubsection{Single point and insertion mutation}

Mutation will effect on new children by changing the number of genes in each new chromosome PL*Pm. Where PL is the length of the chromosome and $\mathrm{Pm}$ is the mutation probability. Single point mutation and insertion mutation will be merged to not only change arrangement genes in the chromosome but also replace the gene value by a random value between 0 and 255 to ensure that a new gray level in new children that does not exist in its parents.

\subsubsection{Replacement}

The final step is to replace parents by new children to confirm a new generation.

\subsubsection{Terminating criteria}

Terminating criteria is a condition that is used to stop the GA procedure. This condition can be a specific number of generations, timing constraint, etc. Two termination criteria were considered. The first is when the difference of best fitness in two consecutive generations is less than a certain " $\varepsilon$ " and this condition is repeated two times when running the algorithm. Where the value of " $\varepsilon$ " were chosen as" 0.01 *best fitness" (the best fitness is the fitness of the last generation). The second terminating factor is reaching a given maximum number of generations.

So, components are common to propose a value that ranges between 0.4 and 0.9 for the crossover probability. So $c$ (Crossover probability) of 0.8 was adopted in this research. The Mutation probability between 0.001 and 0.01 is given because a high mutation probability would change GA to random search. So $\mathrm{m}$ (Mutation probability) of 0.01 was adopted in this research. A maximum number of generations is 100 , and the number of population is 10 .

\section{ANALYSIS OF RESULTS}

To demonstrate the performance of the proposed algorithm, the proposed method provided by MatLab 2016. Several experiments were conducted to evaluate the performance of the proposed algorithm. These experiments were tested on some bench mark images of a size $256 * 256$. Also, the proposed method is applied on real cloudy images taken by LandSat8.

The performance of the proposed technique was contrasted against the other three methods as HE (Histogram equalization) [2], Contrast Stretching [2] and the genetic method [8].

The algorithms were tested and compared according to two

Table 1 A comparative study of contrast enhancement methods applied to grey cloudy images and based upon the number of edges

\begin{tabular}{|c|c|c|c|c|}
\hline Image & $\begin{array}{c}\text { Proposed } \\
\text { method }\end{array}$ & HE & $\begin{array}{c}\text { Contrast } \\
\text { stretching }\end{array}$ & $\begin{array}{c}\text { GA in } \\
{[\mathbf{8}]}\end{array}$ \\
\hline Tornado & 2410 & 1887 & 1740 & 2500 \\
\hline Plane & 3355 & 3260 & 2266 & 3320 \\
\hline Crowd & 3515 & 3327 & 3408 & 3495 \\
\hline Galaxia & 1890 & 1347 & 1469 & 1964 \\
\hline
\end{tabular}

Table 2 A comparative study of contrast enhancement methods applied to grey cloudy images and based upon the PSNR

\begin{tabular}{|c|c|c|c|c|}
\hline Image & $\begin{array}{c}\text { Proposed } \\
\text { method }\end{array}$ & HE & $\begin{array}{c}\text { Contrast } \\
\text { stretching }\end{array}$ & $\begin{array}{c}\text { GA in } \\
\text { [8] }\end{array}$ \\
\hline Tornado & 16.80 & 12.81 & 12.45 & 12.09 \\
\hline Plane & 17.22 & 13.50 & 14.40 & 14.21 \\
\hline Crowd & 20.88 & 12.98 & 11.84 & 16.46 \\
\hline Galaxia & 17.16 & 11.52 & 12.34 & 14.23 \\
\hline
\end{tabular}

evaluation criterions; a number of edges and the PSNR (Peak Signal-to-Noise Ratio) of images.

The number of detected edges for each of the output images is obtained by the known Sobel edge detector. The number of edges is used as a factor for evaluating the detailed content level of the resulted images. The images with the highest number of edges are rated as having high detailed contents [5].

Also, Peak Signal-to-Noise Ratio (PSNR) is a mathematical measure of image quality based on the pixel difference between two images [10]. The PSNR measure is an estimate of the quality of the enhanced image compared with the original image.

Table 1 summarizes a comparative study of the number of edges in images enhanced by the proposed method against HE (Histogram Equalization), Contrast Stretching, and the genetic algorithm [8]. For comparing, the algorithms are applied on a sample of gray benchmark images. Another factor for evaluating the quality of images PSNR is presented in Table 2 .

The following Figure 5 and 6 show the results of tables 1 and 2 respectively in a column chart representation. 


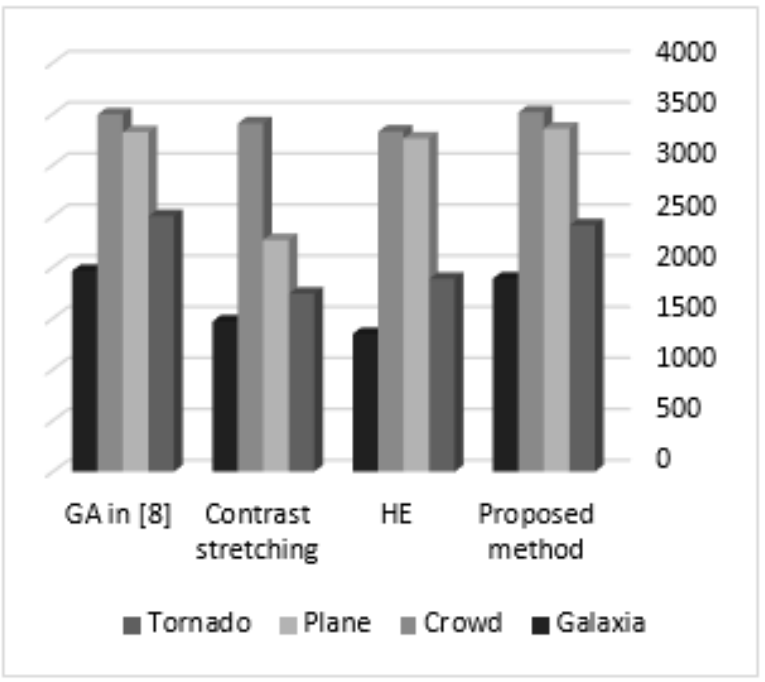

Fig 5: A comparative chart of contrast enhancement methods applied to grey cloudy images and based upon the number of edges

Figure 5 shows that the number of edges for the proposed method is not always greater than the number of edges for other corresponding enhancement algorithms. The proposed contrast fitness function is affected on choosing the enhanced image by the contrast relative difference. Thus in some cases, the images with the higher number of edges are not chosen as output enhanced image. The number of edges in the image after using the other corresponding enhancement algorithms is sometimes greater than that after enhancing by the proposed method. This happens in the case of the clear images. And Figure 6 shows that the PSNR in the image after enhancing by the proposed method is always greater. So the image after enhancement by the proposed method is always better than the corresponding values in the other enhancement algorithms. As shown in following groups of images in figures 7, 8, 9 and 10.

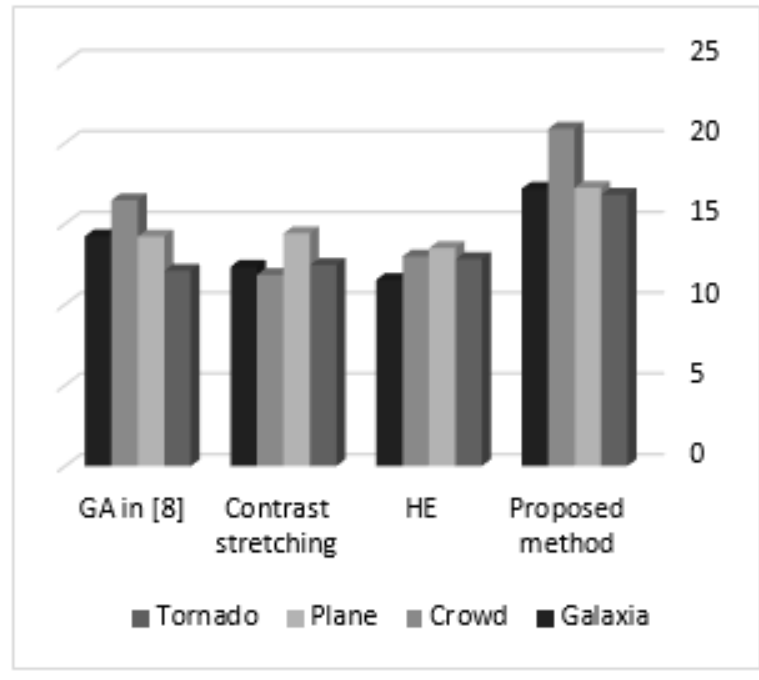

Fig 6: A comparative chart of contrast enhancement methods applied to grey cloudy images and based upon the PSNR

To implement the proposed method on color images the color image is divided to individual bands and get the summation of fitness values of each band individually. The following figures 11, 12 and 13 show some color images that are enhanced by the proposed method, HE (Histogram equalization), Contrast Stretching, and the genetic Algorithm in [8].

The proposed method shows effectiveness to enhance noncloudy images. It can also be implemented to enhance the cloudy images by cloud removal as a preprocessing step. Cloud removal is very useful especially in satellite images because it gives us the ability to use any satellite image even if it is cloudy. The following figures show a real example of a cloudy satellite image before and after enhancement by the proposed method. The group of images is shown in figure 11 .
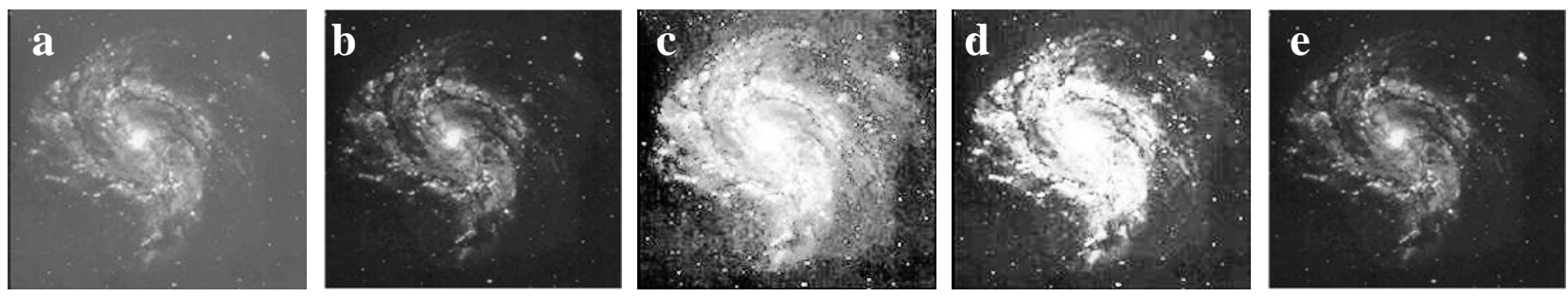

Fig 7: (a) Original Tornado image; the result of the enhancement by the following methods (b) proposed method; (c) HE;

(d) Contrast stretching (e) GA in [8]
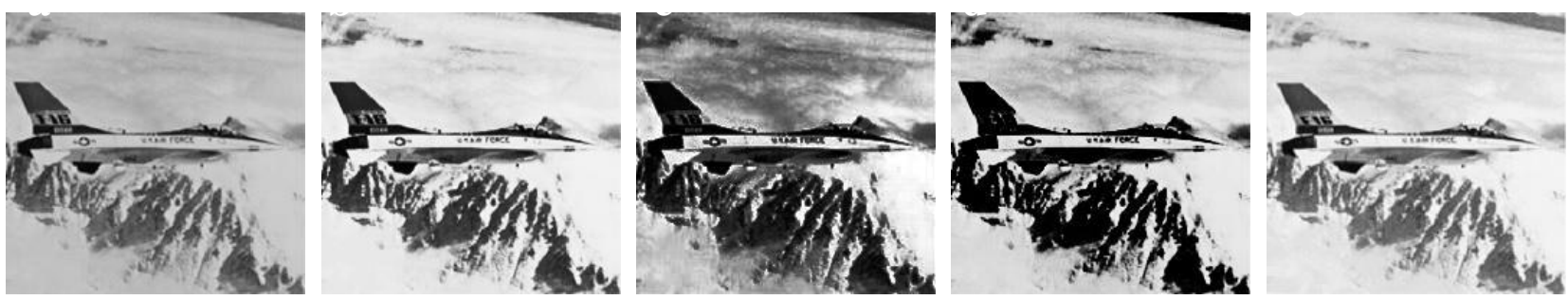

Fig 8: (a) Original plane image; the result of the enhancement by the following algorithms (b) proposed method; (c) HE;

(d) Contrast stretching (e) GA in [8] 

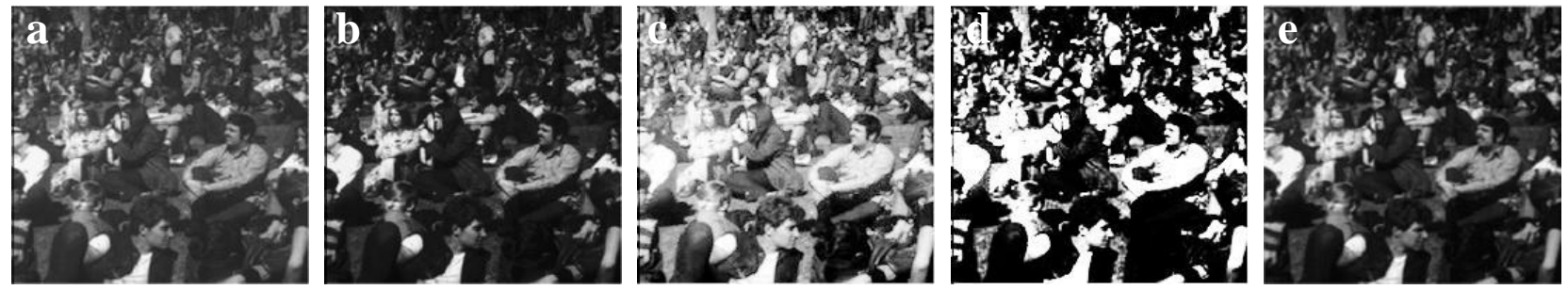

Fig 9: (a) Original crowd image; the result of the enhancement by the following methods (b) proposed method; (c) HE; (d) Contrast stretching (e) GA in [8]
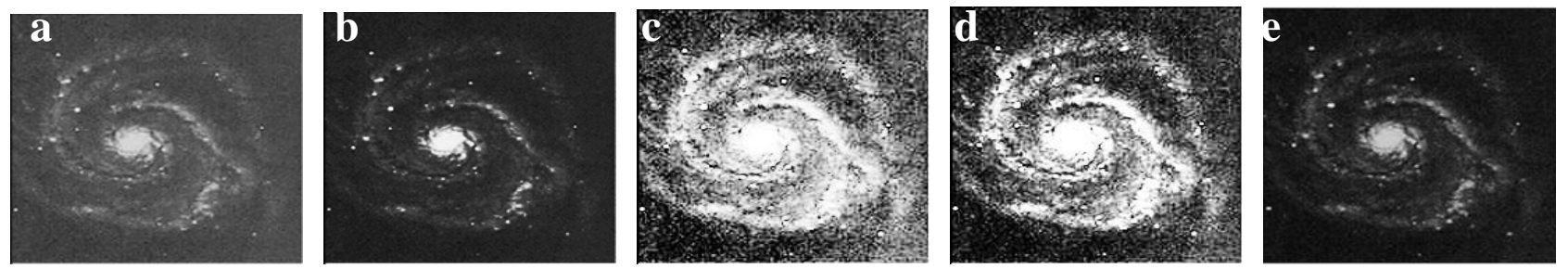

Fig 10: (a) Original galaxia image; the result of the enhancement by the following methods (b) proposed method; (c) HE;(d) Contrast stretching (e) GA in [8]
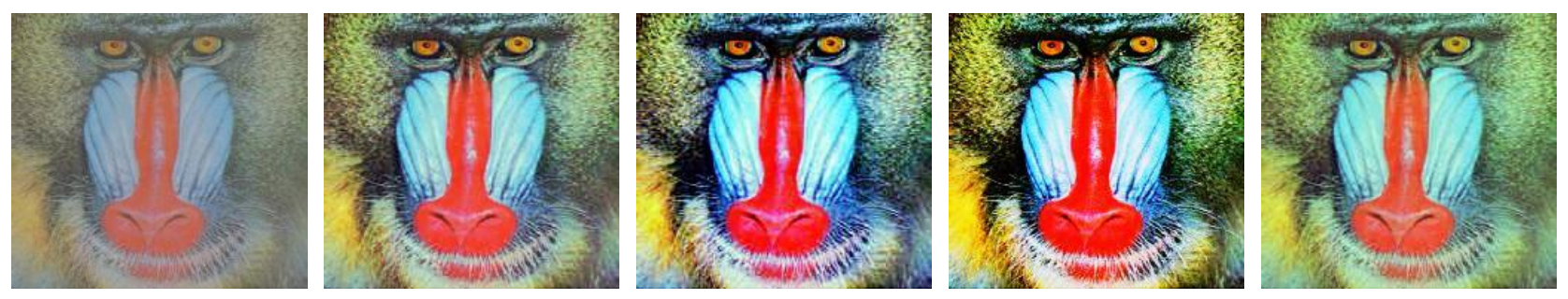

Fig 11: (a) Original monkey image; the result of the enhancement by the following methods (b) proposed method; (c) HE;

(d) Contrast stretching (e) GA in [8]
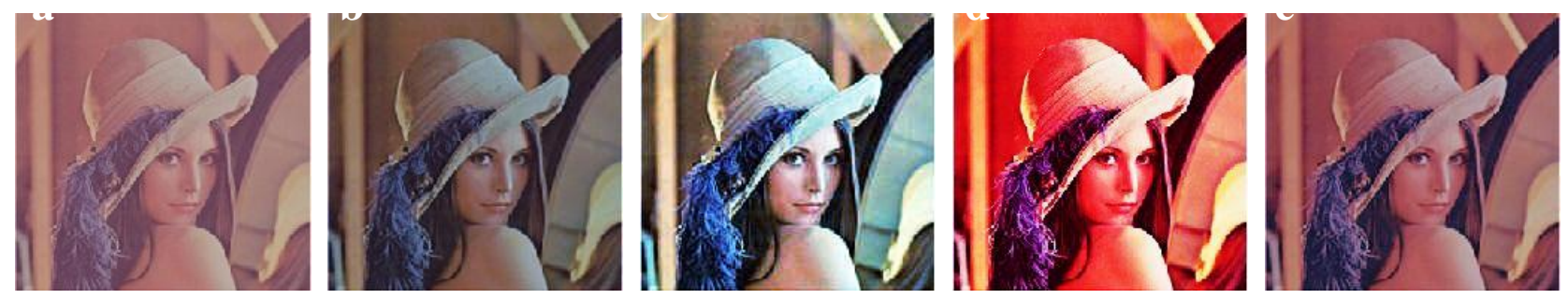

Fig 12: (a) Original lenda image; the result of the enhancement by the following methods (b) proposed method; (c) HE;

a

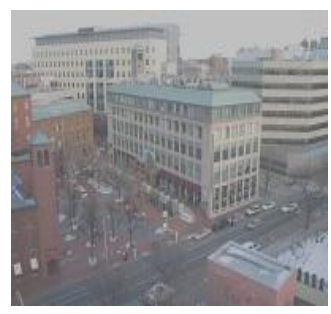

b

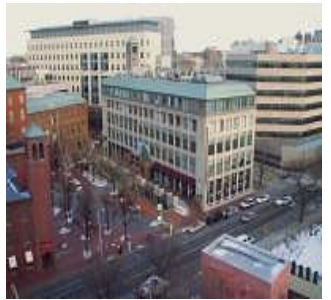

(d) Contrast stretching (e) GA in [8]
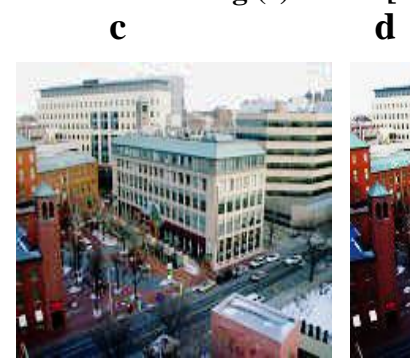

d

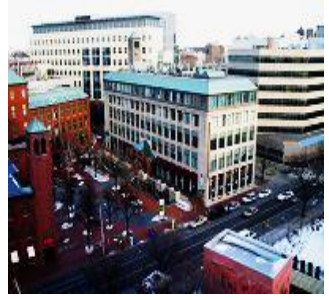

e

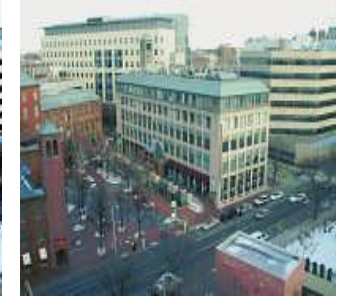

Fig 13: (a) Original low contrast image; the result of the enhancement by the following methods (b) proposed method; (c) HE; (d) Contrast stretching (e) GA in [8] 

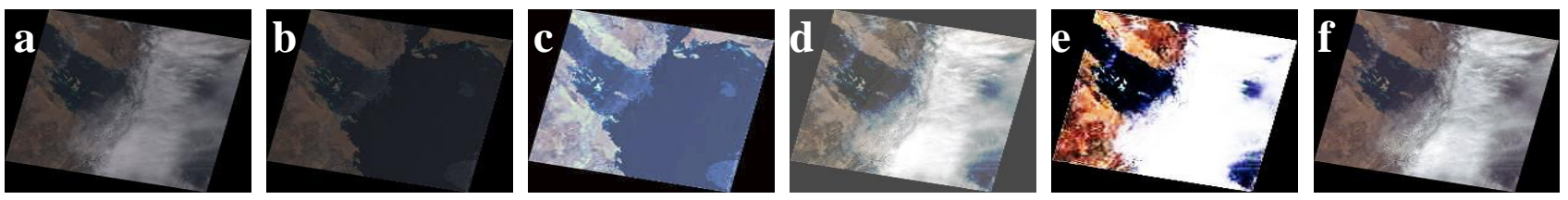

Fig 14: (a) Original Ras Mohamed image; (b) The result of preprocess cloud removal; the result of the enhancement by the following methods (c) proposed method; (d) HE; (e) Contrast stretching (f) GA in [8]

Table 3 summarizes comparative study of the number of edges in colored and cloudy images of separated three colored bands enhanced by the proposed method against HE (Histogram equalization), Contrast Stretching, and the genetic Algorithm in [8].

Table 2 A comparative study of contrast enhancement methods applied to colored and cloudy images and based upon the number of edges

\begin{tabular}{|c|c|c|c|c|c|c|c|c|c|c|c|c|}
\hline \multirow[b]{2}{*}{ Image } & \multicolumn{3}{|c|}{ Proposed GA } & \multicolumn{3}{|c|}{ HE } & \multicolumn{3}{|c|}{ Contrast stretching } & \multicolumn{3}{|c|}{ GA in [8] } \\
\hline & Band 2 & Band 3 & Band 4 & Band 2 & Band 3 & Band 4 & Band 2 & Band 3 & Band 4 & Band 2 & Band 3 & Band 4 \\
\hline Monkey & 1360 & 1484 & 1491 & 1502 & 1515 & 1548 & 1527 & 1679 & 1624 & 1469 & 1402 & 1469 \\
\hline Lenda & 1353 & 1376 & 1385 & 1802 & 1554 & 1549 & 1581 & 1417 & 1545 & 1569 & 1564 & 1576 \\
\hline Low contrast & 1623 & 1599 & 1521 & 1540 & 1527 & 1592 & 1900 & 1894 & 1725 & 1590 & 1604 & 1487 \\
\hline Ras Mohamed & 1189 & 1294 & 1331 & 1005 & 1056 & 1144 & 1519 & 1526 & 1482 & 847 & 859 & 866 \\
\hline
\end{tabular}

Table 4 summarizes comparative study of PSNR in color images of separated three colored bands enhanced by the proposed GA against HE (Histogram equalization), Contrast Stretching, and the genetic Algorithm in [8].

Table 1 A comparative study of contrast enhancement methods applied to colored and cloudy images and based upon the PSNR

\begin{tabular}{|c|c|c|c|c|c|c|c|c|c|c|c|c|}
\hline \multirow{2}{*}{ Image } & \multicolumn{3}{|c|}{ Proposed GA } & \multicolumn{3}{c|}{ HE } & \multicolumn{3}{c|}{ Contrast stretching } & \multicolumn{3}{c|}{ GA in [8] } \\
& & & & & & & & \\
& Band 2 & Band 3 & Band 4 & Band 2 & Band 3 & Band 4 & Band 2 & Band 3 & Band 4 & Band 2 & Band 3 & Band 4 \\
& & & & & & & & & & & & \\
\hline Monkey & 22.39 & 17.77 & 22.23 & 14.87 & 13.75 & 14.63 & 12.45 & 13.00 & 13.15 & 21.52 & 16.91 & 21.52 \\
\hline Lenda & 22.68 & 19.85 & 19.52 & 13.05 & 14.41 & 13.19 & 12.67 & 12.61 & 12.41 & 21.15 & 19.26 & 18.91 \\
\hline Low contrast & 20.13 & 19.66 & 18.69 & 14.96 & 15.21 & 14.96 & 12.03 & 11.74 & 12.22 & 19.51 & 18.88 & 17.49 \\
\hline Ras Mohamed & 10.33 & 10.56 & 11.75 & 6.60 & 6.37 & 6.39 & 5.38 & 5.21 & 4.82 & 9.01 & 8.93 & 8.75 \\
\hline
\end{tabular}

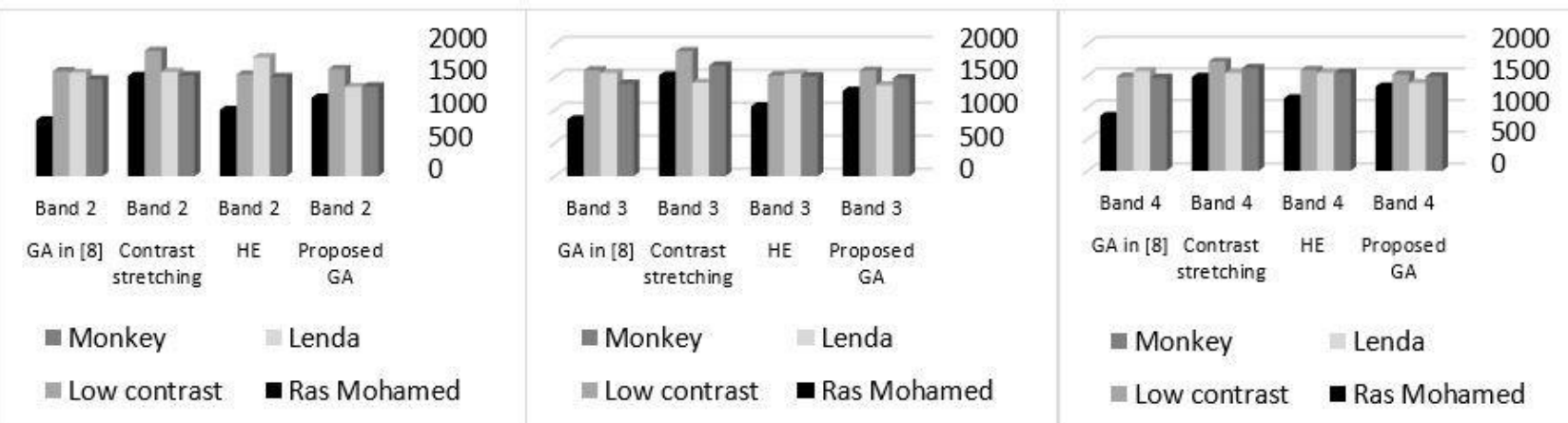

Fig 14: Number of edges of separate three colored bands
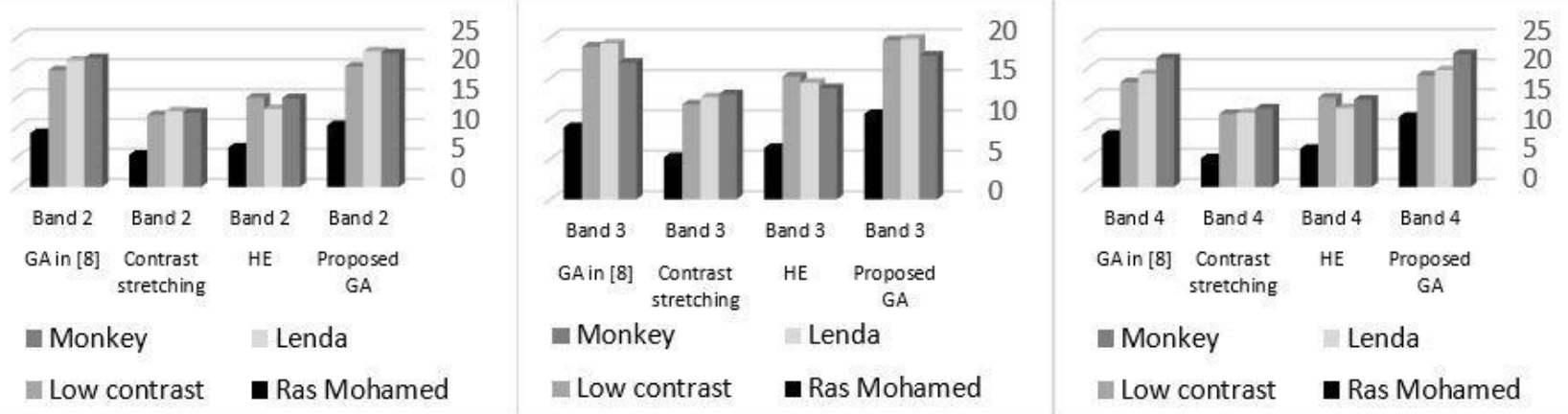

Fig 15: PSNR with separate three colored bands 
Despite the number of edges in the image after Cloud removal is lower than their number in the image before Cloud removal, it does not mean that the image before Cloud removal is better than the image after Cloud removal. But it means that there are some fake edges in the image before Cloud removal as shown in figure 14.

Also, figure 15 presents the comparison of the PSNR in the image after enhancement by the proposed method is always greater than the images resulting from applying the other enhancement algorithms. So the image after enhancement by the proposed method is always better than the other enhancement algorithms as well as the proposed method has a great feature to enhance cloudy images which does not exist in the other enhancement algorithms that can enhance clear images only. The results are presented relative to three colored disjoint bands of a multi-spectra image.

\section{CONCLUSIONS}

In this paper, a method is proposed to enhance the contrast of the cloudy satellite images. The contrast enhancement proposed method is based on genetic algorithm components and closest spectral fit method. Two different criteria have been stressed namely the number of detected edges and the PSNR. The proposed method is compared against Histogram equalization, Contrast stretching and Genetic Algorithm in [8]. But to be fair, the methods are applied on a sample of gray images, colored images, and cloudy colored images. In cloudy colored images, the proposed method's results are better than other methods' results up to $84 \%$. But in other cases, under PSNR criteria, the proposed method is also better than other methods up to $28 \%$. Future extension of this work would be to introduce AI techniques for cloud removal rather than analytic methods. Also, improving the genetic algorithm time complexity is an important issue.

\section{REFERENCES}

[1] Michael Pidwarny (2013). Remote Sensing, The Encyclopedia www.eoearth.org/view/article/155700/

[2] Gonzalez R.C., and Woods R.E., (2009). Digital Image Processing (3rd Ed.). Pearson Prentice Hall.

[3] Golamroza Anbarjavari (2014). Digital Image Processing: Contrast, Enhancement, University of Turtu, https://sisu.ut.ee/imageprocessing/book/

[4] Khushbu Jain, Indra Bhan Arya, (2014) A Survey of Contrast Enhancement Technique for Remote Sensing
Images. International Journal of Electrical. Electronics and Computer Engineering Vol.3, pp.1-6.

[5] Komal R. Hole, Vijay S. Gulhane and Nitin D. Shellokar. (2013) Application of Genetic Algorithm for Image Enhancement and Segmentation. International Journal of Advanced Research in Computer Engineering \& Technology Vol. 2. Issue. 4.

[6] Manikandan S., Ramar K., Willjuice M. Iruthayarajan K. and Srinivasagan G. (2014). Multilevel thresholding for segmentation of medical brain images using real coded genetic algorithm. Elsevier international journal Vol. 47, pp. 558-568.

[7] Yang M., Yang Y., Su T., and Huang K. (2014), an Efficient Fitness Function in Genetic Algorithm Classifier for Land use Recognition on Satellite Images. Hindawi Publishing Corporation Scientific World Journal Vol. 12, pp. 12.

[8] Sara Hashemi, Soheila Kiani, Navid Noroozi, Mohsen Ebrahimi Moghaddam. (2010). An Image Enhancement Method Based On Genetic Algorithm. Pattern Recognition Letters. Vol. 31, pp. 1816-1824.

[9] Yong Wang and Yang Shen. (2015). Removal of thin clouds in visible bands using spectrum characteristics of the visible bands. Geoscience and Remote Sensing Symposium (IGARSS), IEEE International, Milan. pp. $929-932$.

[10] Po-Hung Tsai, Kang-Hua Lai and Jyun-Yuan Chen. (2013) Cloud Removal from Multi temporal Satellite Images Using Information Cloning. Geoscience and Remote Sensing. IEEE Transactions. Vol. 51, pp. 232 241 .

[11] Rosin. (1997). Edges saliency measures and automatic thresholding. Machine Vision and Applications, Springer Vol. 9, pp. 139-159.

[12] Jean-Bernard Martens Lydia Meesters. (1998). Image dissimilarity. Signal Processing, Elsevier Vol. 70, pp. 155- 176

[13] Laboudi Z., and Chikh, Z., (2012). Comparison of Genetic Algorithm and Quantum Genetic Algorithm. The International Arab Journal of Information Technology. Vol. 9, No. 3. 21076. 